\title{
Estimation of Cross-Species Introgression Rates using Genomic Data Despite Model Unidentifiability
}

\author{
Ziheng Yang ${ }^{\mathrm{a}, 1}$ and Tomáš Flouri ${ }^{\mathrm{a}}$ \\ a'Department of Genetics, Evolution and Environment, University College London, Gower Street, London WC1E 6BT, UK
}

This manuscript was compiled on August 14, 2021

\begin{abstract}
The multispecies coalescent with introgression (MSci) model accommodates both the coalescent process and cross-species introgression/hybridization events, two major processes that create genealogical fluctuations across the genome and gene-tree-species-tree discordance. Full likelihood implementations of the MSci model take such fluctuations as a major source of information about the history of species divergence and gene flow, and provide a powerful tool for estimating the direction, timing and strength of cross-species introgression using multilocus sequence data. However, introgression models, in particular those that accommodate bidirectional introgression (BDI), are known to cause unidentifiability issues of the label-switching type, whereby different models or parameters make the same predictions about the genomic data and thus cannot be distinguished by the data. Nevertheless, there has been no systematic study of unidentifiability when full likelihood methods are applied. Here we characterize the unidentifiability of arbitrary BDI models and derive simple rules for its identification. In general, an MSci model with $k$ BDI events has $2^{k}$ unidentifiable towers in the posterior, with each BDI event between sister species creating within-model unidentifiability and each BDI between non-sister species creating cross-model unidentifiability. We develop novel algorithms for processing Markov chain Monte Carlo (MCMC) samples to remove label switching and implement them in the BPP program. We analyze genomic sequence data from Heliconius butterflies as well as synthetic data to illustrate the utility of the BDI models and the new algorithms.
\end{abstract}

Multispecies coalescent | introgression | unidentifiability | BPP | MSci | label-switching

$$
\text { G }
$$
enomic sequences sampled from modern species contain rich historical information concerning species divergences and crossspecies gene flow. In the past two decades, analysis of genomic sequence data has demonstrated the widespread nature of cross-species hybridization or introgression $(1,2)$. A number of statistical methods have been developed to infer introgression using genomic data, most of which use data summaries such as the estimated gene trees (3-5). Full-likelihood methods applied directly to multi-locus sequence alignments (6-8) allow estimation of evolutionary parameters including the timing and strength of introgression, as well as species divergence times and population sizes for modern and extinct ancestral species. These have moved the field far beyond simply testing for the presence of cross-species gene flow.

Models of cross-species introgression are known to cause unidentifiability issues, whereby different introgression models make the same probabilistic predictions about multilocus sequence data, and cannot be distinguished by such data (9-12). If the probability distributions of the data are identical under model $m$ with parameters $\Theta$ and under model $m^{\prime}$ with parameters $\Theta^{\prime}$, with

$$
f(X \mid m, \Theta)=f\left(X \mid m^{\prime}, \Theta^{\prime}\right)
$$

for essentially all possible data $X$, the models are unidentifiable by data $X$. Here we use the term within-model unidentifiability if $m=m^{\prime}$ and $\Theta \neq \Theta^{\prime}$, or cross-model unidentifiability if $m \neq m^{\prime}$. In the former case, two sets of parameter values in the same model are unidentifiable, whereas in the latter, two distinct models are unidentifiable. There have been very limited studies of unidentifiability of introgression models, which examined heuristic methods that use gene tree topologies (either rooted or unrooted) as data (10-12), but the issue has not been studied when full-likelihood methods are applied. Note that unidentifiability depends on the data and the method of analysis. An introgression model unidentifiable given gene tree topologies alone may be identifiable given gene trees with coalescent times. Similarly, a model unidentifiable using heuristic methods may be identifiable when full likelihood methods are applied to the same data. It is thus important to study the problem when full likelihood methods are applied, because unidentifiability by a heuristic method may reflect its inefficient use of information in the data rather than the intrinsic difficulty of the inference problem (13).

Among the different types of MSci models developed (6-8), the bidirectional-introgression (BDI) model (or model D in (8), fig. 1a) is one of the most useful in real data analysis (e.g., 14, 15). The basic BDI model for two species involves nine parameters, with $\Theta=\left(\theta_{A}, \theta_{B}\right.$, $\theta_{X}, \theta_{Y}, \theta_{R}, \tau_{R}, \tau_{X}, \varphi_{X}, \varphi_{Y}$ ) (fig. 1a). Note that an introgression model is similar to a species tree except that there are hybridization nodes representing cross-species introgressions, besides speciation nodes representing species divergences. While a speciation node has one parent and two daughters, a hybridization node has two parents and one daughter. The introgression probabilities $(\varphi$ and $1-\varphi)$ describe the contributions of the two parental populations to the hybrid species. When we trace the genealogical history of a sample of sequences from the modern species backwards in time and reach a hybridization node, each of the sequences takes the two parental paths with probabilities $\varphi$ and $1-\varphi$. There are thus three types of parameters in an introgression (or MSci) model: the times of species divergence and introgression $(\tau \mathrm{s})$, the (effective) population sizes of modern and ancestral species $(\theta \mathrm{s})$, and the introgression probabilities $(\varphi \mathrm{s})$. Both the divergence times $(\tau \mathrm{s})$ and population sizes $(\theta \mathrm{s})$ are measured in the expected number of mutations per site.

The BDI model, in the case of two species (fig. 1), is noted to have an unidentifiability issue (8). Let $\Theta^{\prime}$ be a set of parameters with the same values as $\Theta$ except that $\varphi_{X}^{\prime}=1-\varphi_{X}, \varphi_{Y}^{\prime}=1-\varphi_{Y}$, $\theta_{X}^{\prime}=\theta_{Y}$, and $\theta_{Y}^{\prime}=\theta_{X}$. Then $f(G \mid \Theta)=f\left(G \mid \Theta^{\prime}\right)$ for any gene tree $G$ (fig. 1b\&c). Thus for every point $\Theta$ in the parameter space, there is a 'mirror' point $\Theta^{\prime}$ with exactly the same likelihood. With $\Theta$, the $A$

ZY and TF conceived the research, designed and conducted the experiment, and wrote the paper. No competing interests are declared.

${ }^{2}$ To whom correspondence should be addressed. E-mail: z.yangucl.ac.uk 
sequences take the left (upper) path at $X$ and enter population $R X$ with probability $1-\varphi_{X}$, coalescing at the rate $\frac{2}{\theta_{X}}$, while with $\Theta^{\prime}$, the same $A$ sequences may take the right (horizontal) path and enter population $R Y$ with probability $\varphi_{X}^{\prime}=1-\varphi_{X}$, coalescing at the rate $\frac{2}{\theta_{Y}^{\prime}}=\frac{2}{\theta_{X}}$. The differences between $\Theta$ and $\Theta^{\prime}$ are in the labelling, with 'left' and $X$ under $\Theta$ corresponding to 'right' and $Y$ under $\Theta$ ', but the probabilities involved are the same. The same argument applies to sequences from $B$ going through node $Y$, and to any numbers of sequences from $A$ and $B$ considered jointly. Thus $f(G \mid \Theta)=f\left(G \mid \Theta^{\prime}\right)$ for essentially all $G$. If the priors on $\varphi_{X}$ and $\varphi_{Y}$ are symmetrical, say $\varphi \sim \operatorname{beta}(\alpha, \alpha)$, the posterior density will satisfy $f(\Theta \mid X)=f\left(\Theta^{\prime} \mid X\right)$ for all $X$. Otherwise the "twin towers" may not have exactly the same height.

The situation is very similar to the label-switching problem in Bayesian clustering (16-19). Consider data $X=\left\{x_{i}\right\}$ as a sample from a mixture of two normal distributions, $\mathbb{N}\left(\mu_{1}, 1\right)$ and $\mathbb{N}\left(\mu_{2}, 1\right)$ with the mixing proportions $p_{1}$ and $1-p_{1}$. Let $\Theta=\left(p_{1}, \mu_{1}, \mu_{2}\right)$ be the parameter vector. Then $\Theta^{\prime}=\left(1-p_{1}, \mu_{2}, \mu_{1}\right)$ will have exactly the same likelihood, with $f(X \mid \Theta)=f\left(X \mid \Theta^{\prime}\right)$ for essentially all data $X$. In effect, the labels 'group 1' and 'group 2' are switched between $\Theta$ and $\Theta^{\prime}$.

As an example, we fit the BDI model of figure $2 \mathrm{a}$ to the first 500 noncoding loci on chromosome 1 in the genomic data from three Heliconius butterfly species: H. melpomene, $H$. timareta, and $H$. numata $(14,20)$. Figure 3 a shows the trace plots for parameters $\varphi_{X}$ and $\varphi_{Y}$ from a Markov chain Monte Carlo (MCMC) run. The Markov chain moves between two peaks, centered around $\left(\varphi_{X}, \varphi_{Y}\right)=$ $(0.35,0.1)$ and $(0.65,0.9)$, respectively. In effect, the algorithm is switching between $\Theta$ and $\Theta^{\prime}$ and changing the definition of parameters. This is a label-switching problem, as occurs in Bayesian clustering. The usual practice of estimating parameters by their posterior means (which are 0.54 for $\varphi_{X}$ and 0.62 for $\varphi_{Y}$ in fig. 3a) and constructing the credibility intervals is inappropriate. Indeed the posterior distribution of $\Theta$ is exactly symmetrical with twin towers, and if the chain is run long enough, the posterior means of $\varphi_{X}$ and $\varphi_{Y}$ will be exactly $\frac{1}{2}$. The results are similar when the first 500 exonic loci are analyzed, in which the Markov chain moves between two towers centered around $(0.3,0.1)$ and $(0.7,0.9)$ (fig. S1a).

Unidentifiable models cannot be applied to real data as they are trying to "distinguish the indistinguishable" (10). Results such as those of figures $3 \mathrm{a} \&$ S1a raise two questions. First, are BDI models with more than two species or two BDI events unidentifiable, and what are the rules? Second how do we deal with the problem of label-switching and make the models useful for real data analyses? Those two problems are addressed in this paper. We study the unidentifiability issue of BDI models for an arbitrary number of species with an arbitrary species tree, when a full-likelihood method is applied to multilocus sequence data. It has been conjectured that an MSci model is identifiable by full likelihood methods using data of multilocus sequence alignments if and only if it is identifiable when the data consist of gene trees with coalescent times (8). We make use of this conjecture and consider two BDI models to be unidentifiable if and only if they generate the same distribution of gene trees with coalescent times. We identify general rules for the unidentifiability of the BDI models. We then develop new algorithms for post-processing the MCMC samples generated from a Bayesian analysis under the BDI model to remove the label-switching. Those advancements make the BDI models usable for real data analysis despite their unidentifiability. We use the BPP program to analyze synthetic datasets as well as genomic data from Heliconius butterflies to demonstrate the utility of the BDI models and the new algorithms.

\section{Theory}

The rule of unidentifiability of BDI models. Suppose species $A$ and $B$ exchange migrants at time $\tau_{X}=\tau_{Y}$ through bidirectional introgression (fig. 4). To study the backwards-in-time process of coalescent and introgression, we can treat nodes $X$ and $Y$ as one node, $X Y$. When sequences from $A$ reach node $X Y$, each of them has probability $1-\varphi_{X}$ of taking the left parental path $(R X)$ and probability $\varphi_{X}$ of taking the right parental path $(S Y)$. Similarly when sequences from $B$ reach node $X Y$, they have probabilities $\varphi_{Y}$ and $1-\varphi_{Y}$ of taking the left $(R X)$ and right $(Y S)$ parental paths, respectively. If we swap branches $A$ and $B$, carrying their population size parameters $(\theta)$ and introgression probabilities $(\varphi)$ in the process, the probability density of the gene-trees remains unchanged. Thus the species tree-parameter combinations $(S, \Theta)$ and $\left(S^{\prime}, \Theta^{\prime}\right)$ of figure $4 \mathrm{~b} \& \mathrm{c}$ are unidentifiable. The processes of coalescent and introgression before reaching nodes $A$ and $B$ are identical between $\Theta$ and $\Theta^{\prime}$, as are the processes past nodes $X$ and $Y$. For example, the rule still applies if each of $A$ and $B$ is a subtree, with introgression events inside, or if there are introgression events involving a descendant of $A$ and a descendant of $B$.

In the case of two species, the parental species $R$ and $S$ (fig. 4) are one node, and the species trees $(A, B)$ and $(B, A)$ are the same. As a result, $\Theta$ and $\Theta^{\prime}$ in figure 4 correspond to two sets of parameter values in the same model, so this is a case of within-model unidentifiability. Otherwise the unidentifiability will be cross-model.

Canonical cases of BDI models. Here we study major BDI models to illustrate the rule of unidentifiability and to provide reference for researchers who may apply those models to analyze genomic datasets.

If we add subtrees onto branches $X A, Y B$, or the root branch $R$ in the two-species tree of figure $1 \mathrm{a}$, so that the BDI event remains to be between two sister species, the model will exhibit within-model parameter unidentifiability (fig. S2), just like the basic model of figure 1a.

If the BDI event is between non-sister species, the model exhibits cross-model unidentifiability. Figures $\mathrm{S} 3 \mathrm{a} \& \mathrm{a}^{\prime}$ show a model with a BDI event between cousins, while in figures $S 3 b \& b^{\prime}$, the two species involved in the BDI event are more distantly related.

Figures S4a, b \&c show three models each with a BDI event between non-sister species. In figure $\mathrm{S} 4 \mathrm{a}, X$ and $Y$ are non-sister species on the original binary species tree. In figure S4b\&c, $X$ and $Y$ are non-sister species because there are introgression events involving branches $R X$ and/or $R Y$. In all three cases, there is cross-model unidentifiability, with the twin towers shown in $\mathrm{S} 4 \mathrm{a}^{\prime}, \mathrm{b}^{\prime}, \& \mathrm{c}^{\prime}$.

The case of two non-sister BDI events for three species is illustrated in figure S5. According to our rule, there are four unidentifiable models in the posterior, with parameter mappings shown in figure S5. One way of seeing that the four models are equivalent or unidentifiable is to assume that the introgression probabilities $\left(\varphi_{X}, \varphi_{Y}, \varphi_{Z}\right.$, and $\left.\varphi_{W}\right)$ are all $<\frac{1}{2}$, and then work out the major routes taken when we trace the genealogical history of sequences sampled from modern species. In such cases, all four models of figure S5 predict the following: most sequences from $A$ will take the route $Z R$ at node $Z W$ with probability $1-\gamma$; most sequences from $B$ will take the route $X-W$ at node $X Y$ (with probability $1-\alpha$ ), then the route $W S$ at node $Z W$ (with probability $1-\delta$ ), before reaching $S R$; and most sequences from $C$ will take the route $Y S$ at node $X Y$ (with probability $1-\beta$, before reaching $S R$. Of course the four models are unidentifiable whatever values the introgression probabilities take. Those models have been used to analyze genomic data from Texas horned lizard (Phrynosoma cornutum) (15).

Figure 5 shows two models for five species, each model involving 
three BDI events. In figure 5a, all three BDI events involve sister species, so that there are $2^{3}=8$ unidentifiable within-model towers in the posterior. In figure $5 \mathrm{~b}$, one BDI event involves non-sister species while two involve sister species, so that there are two unidentifiable models, each of which has four unidentifiable within-model towers in the posterior.

In general, if there are $m$ BDI events between sister species and $n$ BDI events between non-sister species, there will be $2^{m}$ unidentifiable models, each having $2^{n}$ within-model unidentifiable towers.

Unidentifiability of double-DBI models. Figure 6 a shows two BDI events between species $A$ and $B$, which occurred at times $\tau_{X}=\tau_{Y}$ and $\tau_{Z}=\tau_{W}$, respectively. To apply the rule of figure 4 , we treat $Z$ and $W$ as one node so that $X$ and $Y$ are considered sister species. There are then four unidentifiable within-model towers in the posterior space, shown as $\Theta_{1}-\Theta_{4}$ in fig. 6 . The parameter mappings are

\begin{tabular}{lcccccccc}
\hline$\Theta$ & $\varphi_{X}$ & $\varphi_{Y}$ & $\theta_{X}$ & $\theta_{Y}$ & $\varphi_{Z}$ & $\varphi_{W}$ & $\theta_{Z}$ & $\theta_{W}$ \\
\hline$\Theta_{1}: \varphi_{X}<\frac{1}{2}, \varphi_{Z}<\frac{1}{2}$ & $\alpha$ & $\beta$ & $\theta_{X}$ & $\theta_{Y}$ & $\gamma$ & $\delta$ & $\theta_{Z}$ & $\theta_{W}$ \\
$\Theta_{2}: \varphi_{X}<\frac{1}{2}, \varphi_{Z}>\frac{1}{2}$ & $\alpha$ & $\beta$ & $\theta_{X}$ & $\theta_{Y}$ & $1-\gamma$ & $1-\delta$ & $\theta_{W}$ & $\theta_{Z}$ \\
$\Theta_{3}: \varphi_{X}>\frac{1}{2}, \varphi_{W}<\frac{1}{2}$ & $1-\alpha$ & $1-\beta$ & $\theta_{Y}$ & $\theta_{X}$ & $\delta$ & $\gamma$ & $\theta_{W}$ & $\theta_{Z}$ \\
$\Theta_{4}: \varphi_{X}>\frac{1}{2}, \varphi_{W}>\frac{1}{2}$ & $1-\alpha$ & $1-\beta$ & $\theta_{Y}$ & $\theta_{X}$ & $1-\delta$ & $1-\gamma$ & $\theta_{Z}$ & $\theta_{W}$ \\
\hline
\end{tabular}

In general, with $k$ BDI events between two species, which occurred at different time points in the past, there will be $2^{k}$ unidentifiable within-model towers in the posterior. There may be little information in practical datasets to estimate so many parameters: if all sequences have coalesced before they reach the ancient introgression events near the root of the species tree, the introgression probabilities $(\varphi s)$ and the associated population sizes $(\theta \mathrm{s})$ will be nearly impossible to estimate. Thus we do not consider more than two BDI events between two species. Note that even the model with one BDI event is not identifiable by heuristic methods that use gene tree topologies only. A small simulation is conducted to illustrate the feasibility of applying the double-BDI model (fig. 6) to genomic datasets; see Results.

Addressing unidentifiability issues and difficulties with identifiability constraints. According to our rule, MSci models with BDI events can create both within-model and cross-model unidentifiability. Cross-model unidentifiability is relatively simple to identify and deal with. If the MCMC is run with the MSci model fixed (8), only one of the models (e.g., model $S_{1}$ with parameters $\Theta_{1}$ in fig. S5) is visited in the chain. One can then summarize the posterior distribution for parameters under that model (which may be smooth and singlemoded), and the posterior summary may be mapped onto the other unidentifiable models according to the rule. See ref. (15) for such an application of BDI models of figure S5. If the MCMC is trans-model and visits different models in the chain $(6,7)$, the posterior space is symmetrical between the unidentifiable models (such as models $S_{1}-S_{4}$ of fig. S5). However, such symmetry is unlikely to be achieved in the MCMC sample due to well-known mixing difficulties of trans-model MCMC algorithms. One may choose to focus on one of the models (e.g., $S_{1}$ of fig. S5) and post-process the MCMC sample to map the sample onto the chosen model before producing the within-model posterior summary. Oftentimes the MCMC may explore the withinmodel posterior space very well, despite difficulties of moving from one model to another. In all cases, the researcher has to be aware of the unidentifiable models which are equally good explanations of the data (see Discussion).

Our focus here is on within-model unidentifiability created by BDI events between sister species. When there are multiple modes in the posterior, each mode may offer a sensible interpretation of the data, but it is inappropriate to merge MCMC samples from different modes, or to construct posterior summaries such as the posterior means and CIs using MCMC samples that traverse different modes. It is instead more appropriate to summarize the samples for each mode.

A common strategy for removing label-switching is to apply socalled identifiability constraints. In the simple BDI model of figure 1, any of the following constraints may be applicable: $\varphi_{X}<\frac{1}{2}, \varphi_{Y}<\frac{1}{2}$, and $\theta_{X}<\theta_{Y}$. Such identifiability constraints may be imposed during the MCMC or during post-processing of the MCMC samples. As discussed previously $(17,18)$, such a constraint may be adequate if the posterior modes are well separated, but may not work well otherwise. For example, when $\varphi_{X}$ is far away from $\frac{1}{2}$ in all MCMC samples, it is simple to process the MCMC sample to impose the constraint $\varphi_{X}<\frac{1}{2}$. This is the case in analyses of the large datasets in this paper, for example, when all noncoding and exonic loci from chromosome 1 of the Heliconius data are analyzed (table 1). However, when the posterior modes are not well-separated (either because the true parameter value is close to the boundary defined by the inequality or because the data lack information so that the CIs are wide), different identifiability constraints can lead to very different parameter posteriors (16), and an appropriate constraint may not exist. A serious problem in such cases is that imposing an identifiability constraint may generate posterior distributions over-represented near the boundary, with seriously biased posterior means $(17,18)$. For example, $\varphi_{X}$ may have substantial density mass both below and above $\frac{1}{2}$, and imposing the constraint $\varphi_{X}<\frac{1}{2}$ will artificially generate high density mass close to $\varphi_{X}=\frac{1}{2}$. Similarly the posterior distributions of $\theta_{X}$ and $\theta_{Y}$ may overlap, so that the constraint $\theta_{X}<\theta_{Y}$ may not be appropriate.

New algorithms to process MCMC samples from the BDI model to remove label switching. One approach to dealing with labelswitching problems in Bayesian clustering is relabelling. The MCMC is run without any constrain, and the MCMC sample is then postprocessed to remove label-switching, by attempting to move each point in the MCMC sample to its alternative unidentifiable positions in order to, as far as possible, make the marginal posterior distributions smooth and unimodal $(17,18)$. The processed sample is then summarized to generate the posterior of the parameters. Here we follow this strategy and implement three relabelling algorithms for use with the BDI model.

Let $\Theta=\left(\varphi_{X}, \varphi_{Y}, \theta_{X}, \theta_{Y}\right)$, which has a mirror point $\Theta^{\prime}=$ $\left(\varphi_{X}^{\prime}, \varphi_{Y}^{\prime}, \theta_{X}^{\prime}, \theta_{Y}^{\prime}\right)=\left(1-\varphi_{X}, 1-\varphi_{Y}, \theta_{Y}, \theta_{X}\right)$ (fig. 1). The other parameters in the model are not involved in the unidentifiability and are simply copied along. Let $\Theta_{t}, t=1, \cdots, N$, be the $N$ samples of parameters generated by the MCMC algorithm. Each sample is a point in the 4-D space. Let $\sigma_{t}$ be a transform for point $t$, with $\sigma_{t}\left(\Theta_{t}\right)=\Theta_{t}$ to be the original point, and $\sigma_{t}\left(\Theta_{t}\right)=\Theta^{\prime}$ to be the transformed or mirror point (fig. $1 \mathrm{~b} \& \mathrm{c}$ ). With a slight abuse of notation, we also treat $\sigma_{t}$ as an indicator, with $\sigma_{t}=0$ and 1 representing $\Theta_{t}$ and $\Theta_{t}^{\prime}$, respectively. For each sample $t$, we choose either the original point or its mirror point, to make the posterior of the parameters look smooth and single-moded as far as possible. The first two algorithms, called center-of-gravity algorithms $\mathrm{CoG}_{0}$ and $\mathrm{CoG}_{N}$, loop through two steps.

Algorithms $\mathbf{C o G}_{0}$ and $\mathbf{C o G}_{N}$. Initialize. For each point $t, t=$ $1, \cdots, N$, pick either the original point $\left(\Theta_{t}\right)$ or its mirror point $\left(\Theta_{t}^{\prime}\right)$. We set $\sigma_{t}$ to 0 (for $\Theta_{t}$ ) if $\varphi_{X}<\frac{1}{2}$ or $\varphi_{Y}<\frac{1}{2}$, or to 1 (for $\Theta_{t}^{\prime}$ ) otherwise.

- Step 1. Determine the center of gravity, given by the sample means of the parameters, $\mu=\left(\bar{\varphi}_{X}, \bar{\varphi}_{Y}, \bar{\theta}_{X}, \bar{\theta}_{Y}\right)$.

- Step 2. For each point $t=1, \cdots, N$, compare the current and its mirror positions and choose the one closer to the center of 
gravity $(\mu)$.

In step 2, we use the Euclidean distance

$$
d_{0}(\Theta, \mu)=\left[\sum_{j}^{4}\left(\phi_{j}-\mu_{j}\right)^{2}\right]^{1 / 2},
$$

where $\phi_{j}$ are the four parameters in $\Theta: \varphi_{X}, \varphi_{Y}, \theta_{X}, \theta_{Y}$. This is algorithm $\mathrm{CoG}_{0}$.

If we consider different scales in the different dimensions (for example, $\varphi_{X}$ and $\theta_{X}$ may have very different posterior variances), we can calculate the sample variances $v$ (in addition to the sample means $\mu$ ) in step 1 and use them as weights to normalize the differences in step 2, with

$$
d_{N}(\Theta, \mu)=\left[\sum_{j}^{4} \frac{1}{v_{j}}\left(\phi_{j}-\mu_{j}\right)^{2}\right]^{1 / 2} .
$$

We refer to this as algorithm $\mathrm{CoG}_{N}$.

The third algorithm, called the $\beta-\gamma$ algorithm, follows the relabelling algorithm in ref. (18) for Bayesian clustering. We use maximum likelihood (ML) to fit the sample $\left\{\Theta_{t}\right\}$ to independent beta distributions for $\varphi_{X}$ and $\varphi_{Y}$ and gamma distributions for $\theta_{X}$ and $\theta_{Y}$ :

$$
\begin{aligned}
f(\Theta ; \omega)=b\left(\varphi_{X} ; p_{X}, q_{X}\right) \cdot b\left(\varphi_{Y} ; p_{Y}, q_{Y}\right) \\
\times \\
\times g\left(\theta_{X} ; a_{X}, b_{X}\right) \cdot g\left(\theta_{Y} ; a_{Y}, b_{Y}\right),
\end{aligned}
$$

where

$$
\begin{aligned}
& b(\phi ; p, q)=\frac{1}{B(p, q)} \phi^{p-1}(1-\phi)^{q-1}, \\
& g(\phi ; a, b)=\frac{b^{a}}{\Gamma(a)} \phi^{a-1} \mathrm{e}^{-b \phi}
\end{aligned}
$$

are the beta and gamma densities and where $\omega=\left(p_{X}, q_{X}, p_{Y}, q_{Y}\right.$, $\left.a_{X}, b_{X}, a_{Y}, b_{Y}\right)$ is the vector of hyper-parameters.

The log likelihood, as a function of the hyper-parameters $\omega$ and the transforms $\sigma=\left\{\sigma_{t}\right\}$, is

$$
\ell(\omega, \sigma)=\sum_{t}^{N} \ell_{t}\left(\omega, \sigma_{t}\left(\Theta_{t}\right)\right)=\sum_{t}^{N} \log f\left(\sigma_{t}\left(\Theta_{t}\right) ; \omega\right) .
$$

We have implemented the following iterative algorithm to estimate $\omega$ and $\sigma$ by maximizing $\ell$.

Algorithm $\beta-\gamma$. Initialize $\sigma_{t}, t=1, \cdots, N$. As before, we set $\sigma_{t}$ to 0 (for $\Theta_{t}$ ) if $\varphi_{X}<\frac{1}{2}$ or $\varphi_{Y}<\frac{1}{2}$, or to 1 (for $\Theta_{t}^{\prime}$ ) otherwise.

- Step 1. Choose $\hat{\omega}$ to maximize the $\log$ likelihood $\ell$ (eq. 7) with the transforms $\sigma$ fixed.

- Step 2. For $t=1, \cdots, N$, choose $\sigma_{t}$ to maximize $\ell_{t}\left(\hat{\omega}, \sigma_{t}\left(\Theta_{t}\right)\right)$ with the hyper-parameters $\omega$ fixed. In other words compare $\Theta_{t}$ and $\Theta_{t}^{\prime}$ and choose the one that better fits the beta and gamma distributions.

Step 1 fits two beta and two gamma distributions by ML and involves four separate 2-D optimization problems. The maximum likelihood estimates (MLEs) of $p$ and $q$ for the beta distribution $b(\phi ; p, q)$ are functions of $\sum_{t} \log \phi_{t}$ and $\sum_{t} \log \left(1-\phi_{t}\right)$, whereas the MLEs of $a$ and $b$ for the gamma distribution $g(\phi ; a, b)$ are functions of $\sum_{t} \phi_{t}$ and $\sum_{t} \log \phi_{t}$. These are simple optimization problems, which we solve using the BFGS algorithm in the PAML program (21). Step 2 involves $N$ independent optimization problems, each comparing two points ( $\sigma_{t}=0$ and 1 ), with $\omega$ fixed. It is easy to see that the algorithm is nondecreasing (that is, the $\log$ likelihood $\ell$ never decreases) and converges, as step 1 involves ML estimation of parameters in the beta and gamma distributions, and step 2 involves comparing two points.

Note that algorithm $\beta-\gamma$ becomes algorithm $\mathrm{CoG}_{N}$ if the beta and gamma densities are replaced by normal densities.

Algorithms $\mathrm{CoG}_{0}, \mathrm{CoG}_{N}$, and $\beta-\gamma$ for the double-BDI model (fig. 6a). Under the double-BDI model, there are four within-model unidentifiable towers, specified by eight parameters (eq. 2). Thus $\sigma_{t}$ takes four values $(0,1,2,3)$. Let $\Theta=\left(\varphi_{X}, \varphi_{Y}, \varphi_{Z}, \varphi_{W}, \theta_{X}, \theta_{Y}, \theta_{Z}, \theta_{W}\right)$. We use the same strategy and fit four beta distributions to the $\varphi s$ and four gamma distributions to the $\theta \mathrm{s}$, with 16 hyper-parameters in $\omega$. We implement the three algorithms $\left(\beta-\gamma, \mathrm{CoG}_{N}\right.$, and $\left.\mathrm{CoG}_{0}\right)$ as before. We prefer the tower in which the introgression probabilities are small and initialize the algorithm accordingly. The transforms $\left(\sigma_{t}\right)$ are as follows (eq. 2)

- $\sigma_{t}=0$ : if the parameters are in $\Theta_{1}$, do nothing.

- $\sigma_{t}=1$ : if in $\Theta_{2}$, let $\varphi_{Z}=1-\varphi_{Z}, \varphi_{W}=1-\varphi_{W}$, and swap $\theta_{Z}$ and $\theta_{W}$.

- $\sigma_{t}=2$ : if in $\Theta_{3}$, let $\varphi_{X}=1-\varphi_{X}, \varphi_{Y}=1-\varphi_{Y}$, swap $\theta_{X}$ and $\theta_{Y}$, swap $\varphi_{Z}$ and $\varphi_{W}$, swap $\theta_{Z}$ and $\theta_{W}$;

- $\sigma_{t}=3$ : if in $\Theta_{4}$, let $\varphi_{X}=1-\varphi_{X}, \varphi_{Y}=1-\varphi_{Y}$, swap $\theta_{X}$ and $\theta_{Y}$, and let $\varphi_{Z}=1-\varphi_{W}$ and $\varphi_{W}=1-\varphi_{Z}$.

The algorithms are implemented in $\mathrm{C}$ and require minimal computation and storage. Processing $5 \times 10^{5}$ samples takes several seconds, mostly spent on reading and writing files. The algorithms are integrated into the BPP program (22) so that MCMC samples from various BDI models are post-processed and summarized automatically. We also provide a stand-alone program in the github repository abacus-gene/bpp-msci-D-process-mcmc.

\section{Results}

Introgression between Heliconius melpomene and $\boldsymbol{H}$. timareta. We fitted the BDI model of figure 2 to the genomic sequence data from three species of Heliconius butterflies: H. melpomene, H. timareta, and $H$. numata $(14,20)$. When we used the first 500 loci, either noncoding or exonic, there was substantial uncertainty in the posterior of $\varphi_{X}$ and $\varphi_{Y}$, and the MCMC jumped between the twin towers, and the marginal posteriors had multiple modes, due to label switching (figs. 3a \& S1a). Post processing of the MCMC sample using the new algorithms led to single-moded marginal posterior distributions (figs. 3b-d \& S1b-d). The three algorithms produced extremely similar results for both datasets. For example, the posterior mean and $95 \% \mathrm{CI}$ for $\varphi_{X}$ from the noncoding data were $0.356(0.026,0.671)$ by $\mathrm{CoG}_{0}, 0.357(0.026,0.674)$ by $\mathrm{CoG}_{N}$, and $0.354(0.022,0.664)$ by $\beta-\gamma$, while those for $\varphi_{Y}$ were $0.103(0.000,0.304)$ by $\mathrm{CoG}_{0}$ and $\mathrm{CoG}_{N}$, and $0.104(0.000,0.306)$ by $\beta-\gamma$.

We then analyzed all the 2592 noncoding and 3023 exonic loci on chromosome 1. With the large datasets, the parameters were better estimated with narrower CIs and the unidentifiable towers were wellseparated. In fact, the MCMC visited only one of the two towers, but that tower was well explored so that multiple runs produced highly consistent results. We started the MCMC with small values for $\varphi_{X}$ and $\varphi_{Y}$, and post-processing the MCMC samples had no effect.

Estimates of all parameters from the small (with $L=500$ ) and large datasets are summarized in table 1 . In the small datasets, the introgression probabilities were $\varphi_{X} \approx 0.354$ (with the CI 0.022-0.664) for the noncoding data and 0.280 (with CI 0.002-0.547) for the coding loci, while $\varphi_{Y}$ was 0.104 (CI $0.000-0.306$ ) for the coding data and 0.116 (CI $0.000-0.318$ ) for the exonic data. When all loci from chromosome 1 were used, $\varphi_{X}$ was 0.124 (with the CI 0.007-0.243) 
for the noncoding data and 0.161 (with CI $0.070-0.264$ ) for the coding loci, while $\varphi_{Y}$ was 0.048 (CI $0.000-0.139$ ) for the coding data and 0.019 (CI 0.000-0.056) for the exonic data. The estimates were similar between the coding and noncoding data, with greater proportions of migrants in $H$. timareta from $H$. melpomene than in the opposite direction. This was so despite the fact that $H$. melpomene had a smaller effective population size than $H$. timareta. Note that $H$. melpomene has a widespread geographical distribution whereas $H$. timareta is restricted to the Eastern Andes; the small $\theta_{M}$ estimates are most likely due to the fact that the $H$. melpomene sample was from a partially inbred strain to avoid difficulties with genome assembly. Estimates of $\theta$ s and $\tau$ s were smaller for the coding loci than for the noncoding loci, due to selective constraint on nonsynonymous mutations.

Estimates of $\varphi_{X}$ and $\varphi_{Y}$ showed large differences between the small and large datasets, but they involved large uncertainties, with the CIs for large datasets mostly inside the CIs for the small datasets. One reason for the differences may be the variable rate of gene flow across the genome or chromosome. Note that $\varphi$ in the MSci model reflects the long-term effects of gene flow and selection purging introgressed alleles, influenced by linkage to gene loci under natural selection.

Analysis of data simulated under the double-BDI model of figure 6 a. We conducted a small simulation to illustrate the feasibility of the double-BDI model (fig. 6), simulating 10 replicate datasets of $L=500,2000$, and 8000 loci. The three algorithms were used to process the MCMC samples, before they were summarized. A typical case is shown in figure 7 for the case of $L=500$. While there are four unidentifiable towers in the 8-D posterior space (eq. 2), the MCMC visited only two of them, with different values for parameters around the $Z W$ BDI event. The dataset of $L=500$ loci are very informative about the parameters for the recent BDI event at node $X Y\left(\varphi_{X}, \varphi_{Y}\right.$, $\theta_{X}, \theta_{Y}$ ), so that these had highly concentrated posteriors with well separated towers. We started the Markov chains with small values (e.g., 0.1 and 0.2 ) for $\varphi_{X}$ and $\varphi_{Y}$, so that the sampled points were all around the correct tower for those parameters. If the chain started with large $\varphi_{X}$ and $\varphi_{Y}$, it would visit a 'mirror' tower. Thus post-processing of the MCMC samples in the case of $L=500$ mostly affected parameters around the BDI event at $Z W\left(\varphi_{Z}, \varphi_{W}, \theta_{Z}, \theta_{W}\right)$. Figure 7 shows the effects on parameters $\varphi_{Z}$ and $\varphi_{W}$ using the $\beta-\gamma$ algorithm. The $\mathrm{CoG}_{0}$ and $\mathrm{CoG}_{N}$ algorithms produced nearly identical results, and all algorithms were effective in removing label switching. The post-processed samples were summarized to calculate the posterior means and the HPD CIs (fig. 8).

At $L=2000$ or 8000 loci, the four towers were well-separated and the MCMC visited only one of them. Applying the post-processing algorithms either had no effect or, in rare occasions, moved all sampled points from another tower.

Posterior means and the $95 \%$ highest-probability-density (HPD) credibility intervals (CI) for all parameters were summarized in figure 8. Parameters around the BDI event at $Z W\left(\varphi_{Z}, \varphi_{W}, \theta_{Z}, \theta_{W}\right)$ are the most difficult to estimate. Nevertheless, with the increase of data size, the CIs for all parameters become smaller, and the posterior means are converging to the true values. Note that while the simulation is conducted using one set of correct parameter values (say, $\Theta_{1}$ of fig. 6), we consider the estimates to be good if they are close to any of the four towers (say, $\Theta_{2}, \Theta_{3}$, or $\Theta_{4}$ ).

Analysis of data simulated with one BDI event with poorly separated modes. We simulated a more challenging dataset for the relabelling algorithms, with $L=500$ loci under the BDI model of figure 1a with parameter values $\left(\varphi_{X}, \varphi_{Y}\right)=(0.7,0.2)$ (see table $S 1$ ). As $\varphi_{X}$ and $\varphi_{Y}$ are not too far away from $\frac{1}{2}$ and the dataset is small, the posterior modes are poorly separated, with considerable mass near $\left(\frac{1}{2}, \frac{1}{2}\right)$. The unprocessed sample from BPP shows two modes for $\varphi_{Y}$, and one mode around $\frac{1}{2}$ for $\varphi_{X}$, with the posterior means at 0.51 for $\varphi_{X}$ and 0.50 for $\varphi_{Y}$, very close to $\frac{1}{2}$ (fig. S6. These are misleading summaries, as the sample is affected by label switching. The three algorithms $\left(\beta-\gamma, \mathrm{CoG}_{N}\right.$, and $\left.\mathrm{CoG}_{0}\right)$ produce similar results, with single-moded posterior, around the mirror tower $\Theta^{\prime}=(0.3,0.8)$. The posterior means for $\varphi_{X}$ are $0.245,0.236$, and 0.235 , for the three algorithms $\left(\beta-\gamma, \mathrm{CoG}_{N}\right.$, and $\left.\mathrm{CoG}_{0}\right)$, and those for $\varphi_{Y}$ are 0.553 , 0.539 , and 0.538 (table $\mathrm{S} 1$ ). The three algorithms have worked well even when the posterior modes are poorly separated.

The parameters involved in the label switching, $\varphi_{X}, \varphi_{Y}, \theta_{X}, \theta_{Y}$ are poorly estimated, due to the difficulty of separating the towers and to influence from the priors. The estimates should improve if more loci are used in the data. Other parameters in the model are all well estimated (table S1).

\section{Discussion}

Identifiability and low information content of MSci models. The identifiability of other MSci models implemented in BPP are simpler. MSci model A is consistent with three different biological scenarios (fig. 9a-c). In scenario $\mathrm{A}_{1}$, two species $S H$ and $T H$ merge to form a hybrid species $H C$, but the two parental species become extinct after the merge. This scenario may be rare. In scenario $\mathrm{A}_{2}$, species $S U X$ contributes migrants to species $T H C$ at time $\tau_{H}$ and has since become extinct or is unsampled in the data. In scenario $\mathrm{A}_{3}, T U X$ is the ghost species. The three scenarios are unidentifiable using genomic data. Model $\mathrm{B}_{1}$ assumes introgression from species $R A$ to $T C$ at time $\tau_{S}=\tau_{H}$ (fig. 9d). This is distinguishable using genetic data from the alternative model $\mathrm{B}_{2}$ in which there is introgression from $R B$ to $S C\left(\mathrm{~B}_{2}\right.$, fig. 9e). Note that models $\mathrm{B}_{1}$ and $\mathrm{B}_{2}$ are both special cases of model $A_{1}$ with different constraints.

We note that there are many parameter settings and data configurations in which some parameters are hard to estimate, because the data lack information about them. For example, ancestral population sizes for short and deep branches in the species tree are hard to estimate, because most sequences sampled from modern species may have coalesced before reaching that population when we trace the genealogy of the sample backwards in time. Similarly, if not many sequences reach a hybridization node, there will be little information in the data about the introgression probabilities at that node. In such case, even if the model is identifiable mathematically, it may be nearly impossible to estimate the parameters with any precision even with large datasets.

In some cases, certain parameters may be very near the boundary of the parameter space, and this may create near unidentifiability with multiple modes in the posterior. For example, the introgression probability may be close to $\varphi=0$ or 1 , or speciation events may have occurred in rapid succession so that the mother and daughter nodes on the species tree have nearly the same age) (see (15) for an example). The MCMC samples around different modes should be summarized separately.

Estimation of introgression probabilities despite unidentifiability. The three algorithms for post-processing MCMC samples under the BDI model produced very similar results in our applications. In particular the simple center-of-gravity algorithms produced results as good as the more elaborate $\beta-\gamma$ algorithm, despite the fact the normal distribution is a poor approximation to the posterior of the introgression probabilities $\left(\varphi_{X}\right.$ and $\left.\varphi_{Y}\right)$. This may be due to the fact that the distributions (or the distance in the CoG algorithms) are used to compare the sampled points with their unidentifiable mirror 
points only, and are not used to directly approximate the posterior distribution of those parameters, which are estimated by using the processed samples. Similarly, while we fit independent distributions for parameters in the algorithms (eq. 6), there is no need to assume independence in the posterior for the algorithms to work. Furthermore, if there exist multiple modes in the posterior that are not due to label-switching, such genuine multimodality will not be removed by the algorithms (18).

A model with a label-switching type of unidentifiability can still be applied in real data analysis. In the clustering problem, the Bayesian analysis may reveal the existence of two groups, in proportions $p_{1}$ and $1-p_{1}$ with means $\mu_{1}$ and $\mu_{2}$, and it may not matter if it cannot decide which group should be called 'group 1'. The twin towers $\Theta$ and $\Theta$ ' under the BDI model of figure 1 constitute a mathematically similar label-switching problem. However, $\Theta$ and $\Theta^{\prime}$ may represent different biological scenarios or hypotheses. Suppose that species $A$ and $B$ are distributed in different habitats (dry for $A$ and wet for $B$, say), and suppose the ecological conditions have changed little throughout the history of the species. $\Theta$ may mean that species $A$ has been in the dry habitat over the whole time period since species divergence at time $\tau_{R}$, while species $B$ has been in the wet habitat, and they came into contact and exchanged migrants at time $\tau_{X}$. In contrast, $\Theta^{\prime}$ may mean that species $A$ was in the wet habitat since species divergence while species $B$ was in the dry habitat, but when they came into contact (at time $\tau_{X}$ ) they nearly replaced each other, switching places, so that today species $A$ is found in the dry habitat while species $B$ in the wet habitat. The two sets of parameters $\Theta$ and $\Theta^{\prime}$ may thus mean different biological hypotheses. The scenario of total replacement may be implausible for most systems, and in our algorithms, we start with the initial conditions $\varphi_{X}<\frac{1}{2}$ and/or $\varphi_{Y}<\frac{1}{2}$ as much as possible When the introgression probabilities are intermediate, the biological interpretations may not be so clear-cut, but unidentifiability exists nevertheless. In the example of figure S6 and table S1, the choice between the two unidentifiable towers $\Theta=\left(\varphi_{X}, \varphi_{Y}\right)=(0.7,0.2)$ and $\Theta^{\prime}=(0.3,0.8)$ may not be easy. Ultimately, genomic data from modern species provide information about the order and timings of species divergences and cross-species introgressions, but not about the geographical locations and ecological conditions in which the divergences and introgressions occurred. Unidentifiable models discussed in this paper are all of this nature. The algorithms we developed in this paper remove label switching in the MCMC sample, but do not remove the unidentifiability of the BDI models. The researcher has to be aware of the unidentifiability or the equally supported explanations of the genomic data.

In the current implementation of BDI models in BPP, each branch in the species tree is assigned its own population size parameter (8). We note that if all species on the species tree are assumed to have the same population size $(\theta)$, unidentifiability persists. However, if we assume that the population size remains unchanged by the introgression event: e.g., $\theta_{X}=\theta_{A}$ and $\theta_{Y}=\theta_{B}$ in figure 1, the model becomes identifiable. The assumption of the same population size before and after a migration event appears to be plausible biologically. It reduces the number of parameters by two for each BDI event, and removes unidentifiability. It may be worthwhile to implement such models. At any rate, the relabelling algorithms we have implemented makes it possible to apply the BDI models to genomic sequence data despite their unidentifiability.

\section{Materials and Methods}

Introgression in Heliconius butterflies. We fitted the BDI model to the genomic sequence data for three species of Heliconius butterflies: H. melpomene, $H$. timareta, and $H$. numata $(23,24)$. The species tree or MSci model assumed is shown in figure 2, with introgression between $H$. melpomene and $H$. timareta. The two species are known to hybridize, although no attempt has yet been made to infer the direction or magnitude of introgression (except for colour-pattern genes) (24). There are 31,166 autosomal noncoding loci and 36,138 autosomal exonic loci, with 2592 noncoding and 3023 exonic loci on chromosome 1. We conducted two sets of analysis, using either the first 500 loci or all the loci on chromosome 1 .

We used gamma priors for the population sizes $(\theta)$ and for the age of the $\operatorname{root}\left(\tau_{0}\right): \theta \sim G(2,400)$ with the mean 0.005 substitution per site, and $\tau \sim$ $G(2,400)$ with mean 0.005 . The introgression probabilities were assigned beta priors $\varphi \sim B(1,1)$, which is the uniform $\mathbb{U}(0,1)$. We used a burn-in of 16000 iterations, and then took $2 \times 10^{5}$ samples, sampling every 5 iterations. Running time on a server with 9 threads of Intel Xeon Gold $6154 \mathrm{CPU}(3.0 \mathrm{GHz})$ was about 1 hour for the small datasets and 10 hours for the large ones.

Convergence of the MCMC algorithms was assessed by checking for consistency between independent runs, taking into account possible labelswitching issues. In the large datasets analyzed in this paper, the MCMC typically visits only one of the unidentifiable towers, but that tower is wellexplored, with the different runs producing highly consistent posterior after label switching is removed. In such cases, reliable inference is possible (cf.:(19)).

Simulation under the double-BDI model. We simulated and analyzed data to under the double-BDI model of figure 6 . We generated gene trees with branch lengths (coalescent times) and sequences under the JC model (25). The parameters used are $\varphi_{X}=0.1, \varphi_{Y}=0.2, \varphi_{Z}=0.2, \varphi_{W}=0.3, \tau_{R}=0.005$, $\tau_{Z}=\tau_{W}=0.0025, \tau_{X}=\tau_{Y}=0.00125, \theta_{R}=\theta_{Z}=\theta_{X}=\theta_{A}=0.005$, and $\theta_{W}=\theta_{Y}=\theta_{B}=0.02$. Each dataset consists of $L=500,2000$ and 8000 loci, with $S=16$ sequences per species per locus, and with the sequence length to be 500 sites. The numbre of replicate datasets is 10 .

The data were then analyzed using BPP under the double-BDI model (fig. 6) to estimate the 14 parameters. We use gamma priors $\tau_{0} \sim G(2,400)$ for the root age with the mean to be the true value (0.005), and $\theta \sim G(2,200)$ with the mean 0.01 (true values are 0.005 and 0.02 ). We used a burn-in of 32,000 iterations, and then took $5 \times 10^{5}$ samples, sampling every 2 iterations. Analysis of each dataset took $\sim 10 \mathrm{hrs}$ for $L=500$ and $\sim 130 \mathrm{hrs}$ for $L=8000$, using 8 threads on a server. The MCMC samples were processed to remove label-switching before they are summarized to approximate the posterior distribution.

ACKNOWLEDGMENTS. We thank James Mallet and Fernando Seixas for providing the genomic datasets for the Heliconius butterflies, and James Mallet for comments on an earlier draft of the paper. This study has been supported by Biotechnology and Biological Sciences Research Council grant (BB/T003502/1) and a BBSRC equipment grant (BB/R01356X/1).

1. RG Harrison, EL Larson, Hybridization, introgression, and the nature of species boundaries. $J$. Hered. 105 (S1), 795-809 (2014).

2. J Mallet, N Besansky, MW Hahn, How reticulated are species? BioEssays 38, 140-149 (2016).

3. JH Degnan, Modeling hybridization under the network multispecies coalescent. Syst. Biol. 67, 786-799 (2018).

4. RAL Elworth, HA Ogilvie, J Zhu, L Nakhleh, Advances in computational methods for phylogenetic networks in the presence of hybridization in Bioinformatics and Phylogenetics. (Springer) Vol. 29, pp. 317-360 (2019)

5. X Jiao, T Flouri, Z Yang, Multispecies coalescent and its applications to infer species phylogenies and cross-species gene flow. Nat. Sci. Rev. (2021) DOI:10.1093/nsr/nwab127.

6. D Wen, L Nakhleh, Coestimating reticulate phylogenies and gene trees from multilocus sequence data. Syst. Biol. 67, 439-457 (2018).

7. C Zhang, HA Ogilvie, AJ Drummond, T Stadler, Bayesian inference of species networks from multilocus sequence data. Mol. Biol. Evol. 35, 504-517 (2018).

8. T Flouri, X Jiao, B Rannala, Z Yang, A Bayesian implementation of the multispecies coalescent model with introgression for phylogenomic analysis. Mol. Biol. Evol. 37, 1211-1223 (2020).

9. Y Yu, JH Degnan, L Nakhleh, The probability of a gene tree topology within a phylogenetic network with applications to hybridization detection. PLoS Genet. 8, e1002660 (2012).

10. F Pardi, C Scornavacca, Reconstructible phylogenetic networks: do not distinguish the indistinguishable. PLoS Comput. Biol. 11, e1004135 (2015).

11. S Zhu, JH Degnan, Displayed trees do not determine distinguishability under the network multispecies coalescent. Syst. Biol. 66, 283-298 (2017).

12. C Solis-Lemus, A Coen, $C$ Ane, On the identifiability of phylogenetic networks under a pseudolikelihood model. ArXive (2020).

13. T Zhu, Z Yang, Complexity of the simplest species tree problem. Mol. Biol. Evol. (2021) DOI: 10.1093/molbev/msab009. 
bioRxiv preprint doi: https://doi.org/10.1101/2021.08.14.456331; this version posted August 15, 2021. The copyright holder for this preprint (which was not certified by peer review) is the author/funder, who has granted bioRxiv a license to display the preprint in perpetuity. It is made available under aCC-BY-NC-ND 4.0 International license.

14. Y Thawornwattana, J Mallet, Z Yang, Complex introgression history of the erato-sara clade of heliconius butterflies. bioRxiv (2021).

15. N Finger, et al., Genome-scale data reveal deep lineage divergence and a complex demographic history in the texas horned lizard (Phrynosoma cornutum) throughout the southwestern and central usa. Genome Biol. Evol. (2021).

16. S Richardson, $\mathrm{P}$ Green, On Bayesian analysis of mixtures with an unknown number of components (with discussions). J. R. Stat. Soc. B 59, 731-792 (1997).

17. G Celeux, M Hurn, $C$ Robert, Bayesian inference for mixture: the label switching problem in COMPSTAT, eds. R Payne, PJ Green. (Physica, Heidelberg), pp. 227-232 (1998).

18. M Stephens, Dealing with label switching in mixture models. J. R. Stat. Soc. B. 62, 795-809 (2000)

19. A Jasra, CC Holmes, DA Stephens, Markov chain Monte Carlo methods and the label switching problem in Bayesian mixture modeling. Stat. Sci. 1, 50-67 (2005).

20. NB Edelman, et al., Genomic architecture and introgression shape a butterfly radiation. Science 366, 594-599 (2019).

21. Z Yang, Paml 4: Phylogenetic analysis by maximum likelihood. Mol. Biol. Evol. 24, 1586-1591 (2007).

22. T Flouri, X Jiao, B Rannala, Z Yang, Species tree inference with BPP using genomic sequences and the multispecies coalescent. Mol. Biol. Evol. 35, 2585-2593 (2018).

3. GC Heliconius, Butterfly genome reveals promiscuous exchange of mimicry adaptations among species. Nature 487, 94-98 (2012).

24. SH Martin, et al., Genome-wide evidence for speciation with gene flow in Heliconius butterflies. Genome Res. 23, 1817-1828 (2013).

25. T Jukes, C Cantor, Evolution of protein molecules in Munro, H.N., ed. Mammalian Protein Metabolism. (Academic Press, New York), pp. 21-123 (1969).
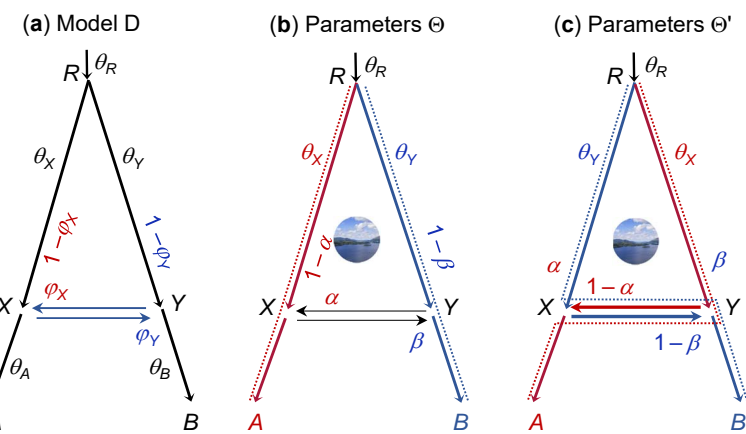

Fig. 1. (a) Bidirectional introgression model or model $D(8)$ assumes introgression in both directions between species $A$ and $B$ at time $\tau_{X}=\tau_{Y}$. (b) and (c) Two sets of parameters $\Theta$ and $\Theta^{\prime}$, with the same parameter values except that $\varphi_{X}^{\prime}=1-\varphi_{X}$ $\varphi_{Y}^{\prime}=1-\varphi_{Y}, \theta_{X}^{\prime}=\theta_{Y}$, and $\theta_{Y}^{\prime}=\theta_{X}$. The dotted lines indicate the main routes taken by sequences sampled from species $A$ and $B$, if both introgression probabilities $\alpha$ and $\beta$ are $\ll \frac{1}{2}$.

(a) 2592 noncoding loci

(b) 3023 exonic loci
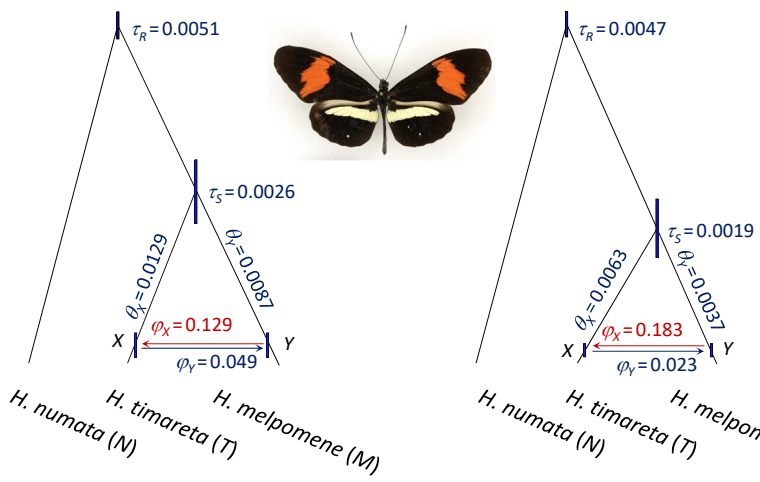

Fig. 2. Species tree and BDI model for Heliconius melpomene, $H$. timareta, and $H$ numata. The branch lengths are drawn to represent the estimated species divergence times (posterior means) using the noncoding and exonic loci from chromosome 1 while the node bars represent the $95 \%$ HPD Cls. See table 1 for estimates of other parameters. Photo of $H$. timareta courtesy of James Mallet. 
bioRxiv preprint doi: https://doi.org/10.1101/2021.08.14.456331; this version posted August 15, 2021. The copyright holder for this preprint (which was not certified by peer review) is the author/funder, who has granted bioRxiv a license to display the preprint in perpetuity. It is made available under aCC-BY-NC-ND 4.0 International license.

Table 1. Posterior means and 95\% HPD Cls (in parenthees) for parameters in the BDI model of figure 2 for the Heliconius data

\begin{tabular}{lcccc}
\hline & Noncoding, $L=500$ & Noncoding, $L=2592$ & Exonic, $L=500$ & Exonic, $L=3023$ \\
\hline$\tau_{R}$ & $4.73(4.33,5.13)$ & $5.10(4.89,5.30)$ & $4.39(3.98,4.81)$ & $4.71(4.54,4.88)$ \\
$\tau_{S}$ & $3.12(2.05,4.19)$ & $2.58(2.12,3.05)$ & $1.95(1.07,2.82)$ & $1.78(1.38,2.19)$ \\
$\tau_{X}=\tau_{Y}$ & $0.62(0.21,1.02)$ & $0.25(0.09,0.40)$ & $0.20(0.03,0.37)$ & $0.13(0.05,0.24)$ \\
$\theta_{M}$ & $1.50(0.62,2.34)$ & $0.69(0.35,1.10)$ & $0.38(0.08,0.70)$ & $0.32(0.14,0.52)$ \\
$\theta_{T}$ & $2.55(1.40,3.74)$ & $1.23(0.65,1.84)$ & $0.79(0.13,1.28)$ & $0.63(0.32,0.94)$ \\
$\theta_{N}$ & $15.1(12.0,18.5)$ & $23.0(20.3,25.7)$ & $11.2(9.11,13.5)$ & $12.4(11.4,13.4)$ \\
$\theta_{R}$ & $5.08(4.12,6.05)$ & $5.74(5.23,6.24)$ & $5.76(4.83,6.70)$ & $6.68(6.24,7.11)$ \\
$\theta_{S}$ & $4.62(1.85,7.40)$ & $6.92(5.48,8.37)$ & $5.31(3.38,7.36)$ & $7.50(6.51,8.49)$ \\
$\theta_{X}$ & $11.4(2.83,21.2)$ & $12.9(7.35,19.6)$ & $8.04(1.67,15.4)$ & $5.80(3.60,8.36)$ \\
$\theta_{Y}$ & $6.78(2.42,11.6)$ & $8.74(5.69,12.0)$ & $4.03(0.60,7.51)$ & $3.49(2.56,4.50)$ \\
$\varphi_{X}$ & $0.354(0.022,0.664)$ & $0.124(0.007,0.243)$ & $0.280(0.002,0.547)$ & $0.161(0.070,0.264)$ \\
$\varphi_{Y}$ & $0.104(0.000,0.306)$ & $0.048(0.000,0.139)$ & $0.116(0.000,0.318)$ & $0.019(0.000,0.056)$ \\
\hline
\end{tabular}

Note.- Estimates of $\tau \mathrm{s}$ and $\theta \mathrm{s}$ are multiplied by $10^{3}$. MCMC samples are processed using the $\beta-\gamma$ algorithm before they are summarized.
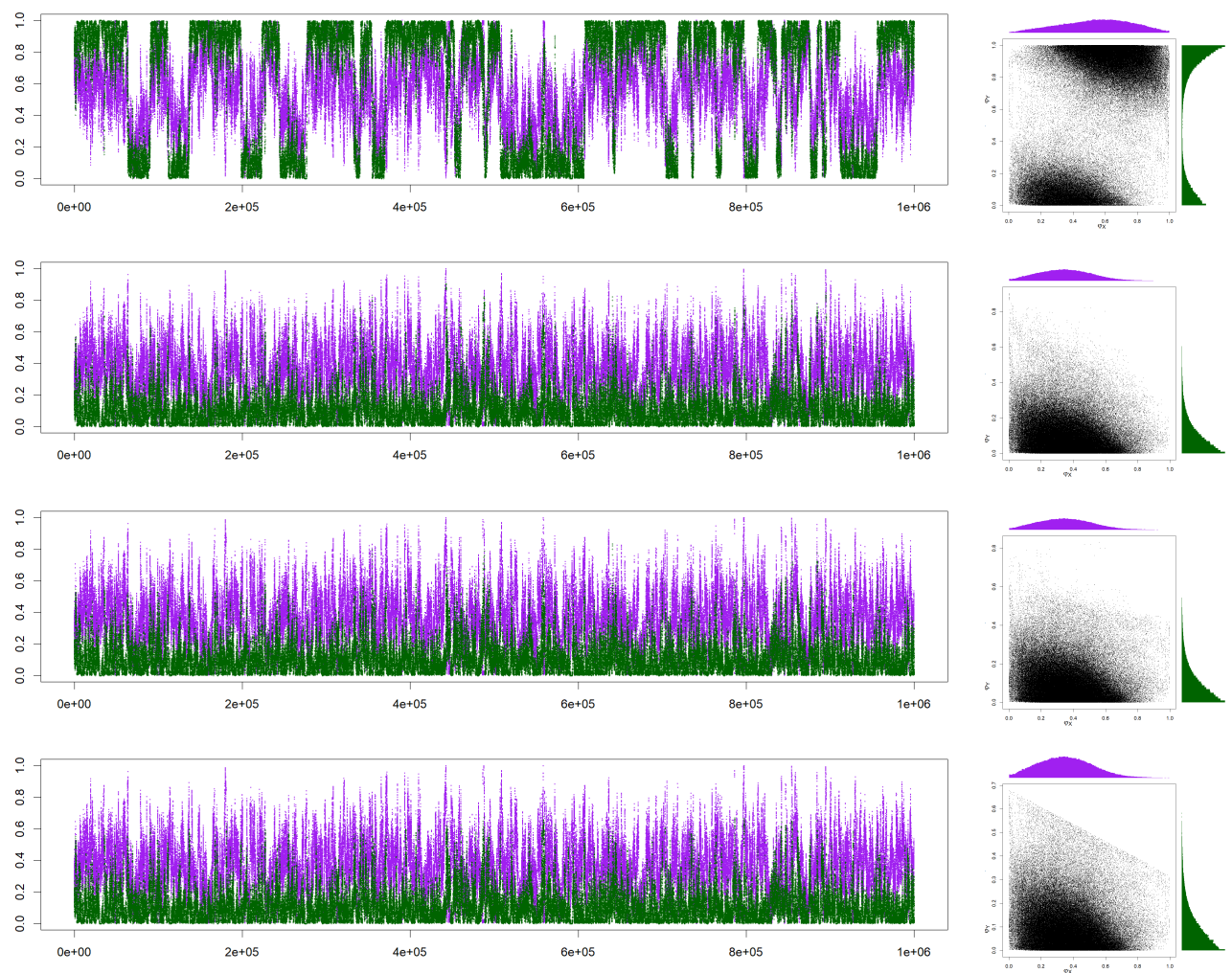

Fig. 3. Trace plots of MCMC samples and 2-D scatter plots for parameters $\varphi_{X}$ (purple) and $\varphi_{Y}$ (green) before (top) and after (bottom three) the post-processing of the MCMC sample in the BPP analysis of the first 500 noncoding loci from chromosome 1 of the Heliconius data under the MSci model of figure 2 . The three algorithms used are $\beta-\gamma$, $\mathrm{CoG}_{N}$, and $\mathrm{CoG}_{0}$. 
bioRxiv preprint doi: https://doi.org/10.1101/2021.08.14.456331; this version posted August 15, 2021. The copyright holder for this preprint (which was not certified by peer review) is the author/funder, who has granted bioRxiv a license to display the preprint in perpetuity. It is made available under aCC-BY-NC-ND 4.0 International license.

(a) BDI

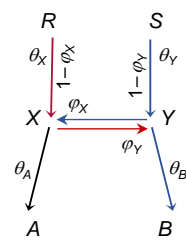

(b) $S, \Theta$

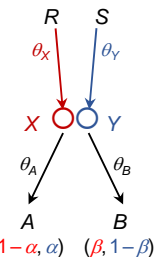

(c) $S^{\prime}, \Theta^{\prime}$

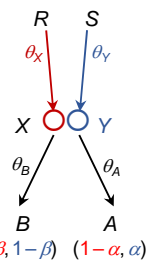

Fig. 4. The rule of BDI unidentifiability. (a) In the BDI model, species $R A$ and $S B$ exchange migrants at time $\tau_{X}=\tau_{Y}$. Treat $X$ and $Y$ as one node with left parent $R X$ with population size $\theta_{X}$ and right parent $S Y$ with population size $\theta_{Y}$. When a sequence from $A$ reaches $X Y$, it takes the left and right parental paths with probabilities $1-\varphi_{X}$ and $\varphi_{X}$, respectively. When a sequence from $B$ reaches $X Y$, it goes left and right with probabilities $\varphi_{Y}$ and $1-\varphi_{Y}$, respectively. (b \& $\mathbf{c}$ ) Placing the two daughters in the order $(A, B)$ as in $\Theta$ or $(B, A)$ as in $\Theta^{\prime}$ does not affect the distribution of gene trees, and constitutes unidentifiable towers in the posterior space. If $X$ and $Y$ are sister species and have the same mother node (with $R$ and $S$ to be the same node), the unidentifiability is within-model; otherwise it is cross-model.

(a) $2^{3}$ towers

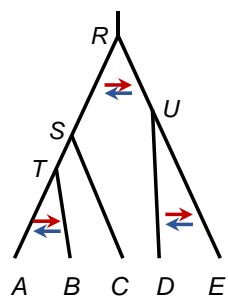

(b) $2^{2}+2^{2}$ towers

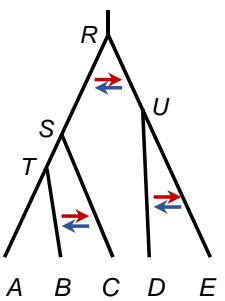

Fig. 5. (a) Three BDI events between sister species creating $2^{3}=8$ within-model towers in the posterior. (b) Two BDI events between sister species and one BDI event between non-sister species creating two unidentifiable models each with four within-model unidentifiable towers in the posterior space.

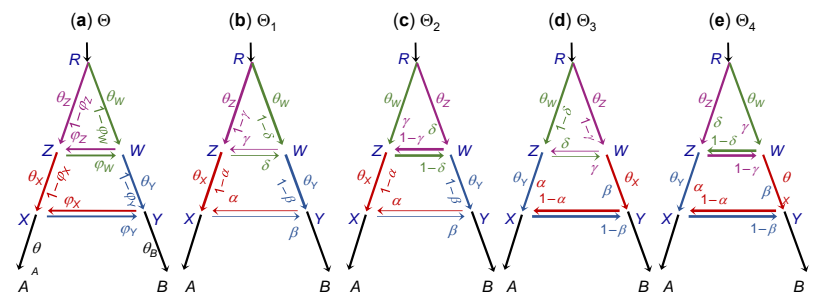

Fig. 6. Double-BDI model between two species $A$ and $B$, with four within-model towers $\left(\Theta_{1}, \Theta_{2}, \Theta_{3}\right.$, and $\left.\Theta_{4}\right)$. (a) The parameters in the model include $7 \theta \mathrm{s}, 3 \tau \mathrm{s}$, and $4 \varphi \mathrm{s}$, with 14 parameters in total. (b)-(e) Four unidentifiable towers showing the mappings of parameters (eq. 2). To apply the rule of figure 4, we treat each pair of BDI nodes as one node, so that $X$ and $Y$ have the same node $Z W$ as the parent, and the unidentifiability caused by the BDI event at nodes $X-Y$ is within-model, as is the unidentifiability for the BDI event at nodes $Z-W$. 
bioRxiv preprint doi: https://doi.org/10.1101/2021.08.14.456331; this version posted August 15,2021 . The copyright holder for this preprint (which was not certified by peer review) is the author/funder, who has granted bioRxiv a license to display the preprint in perpetuity. It is made available under aCC-BY-NC-ND 4.0 International license.
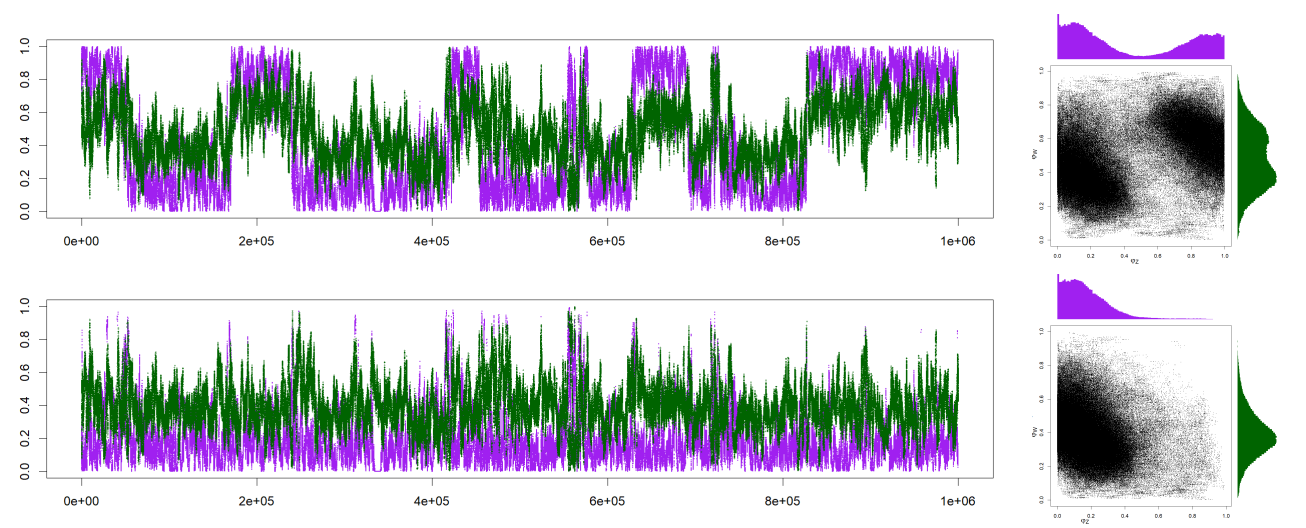

Fig. 7. Trace plots of MCMC samples and 2-D scatter plots for parameters $\varphi_{Z}$ (purple) and $\varphi_{W}$ (green) before (top) and after (bottom) the post-processing of the MCMC samples for the double-DBI model of figure $6 \mathrm{a}$. Post processing used the $\beta-\gamma$ algorithm, while $\mathrm{CoG}_{N}$ and $\mathrm{CoG}_{0}$ produced nearly identical results (not shown). This is for replicate 2 for $L=500$ loci.
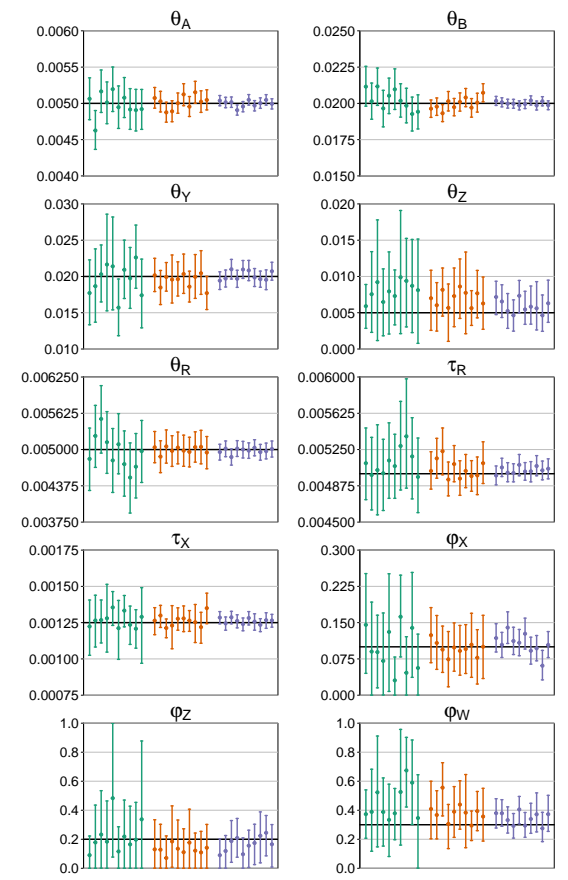

loci $=500-$ loci $=2000-$ loci $=8000$

0.000

$$
\mathrm{i}=8000
$$
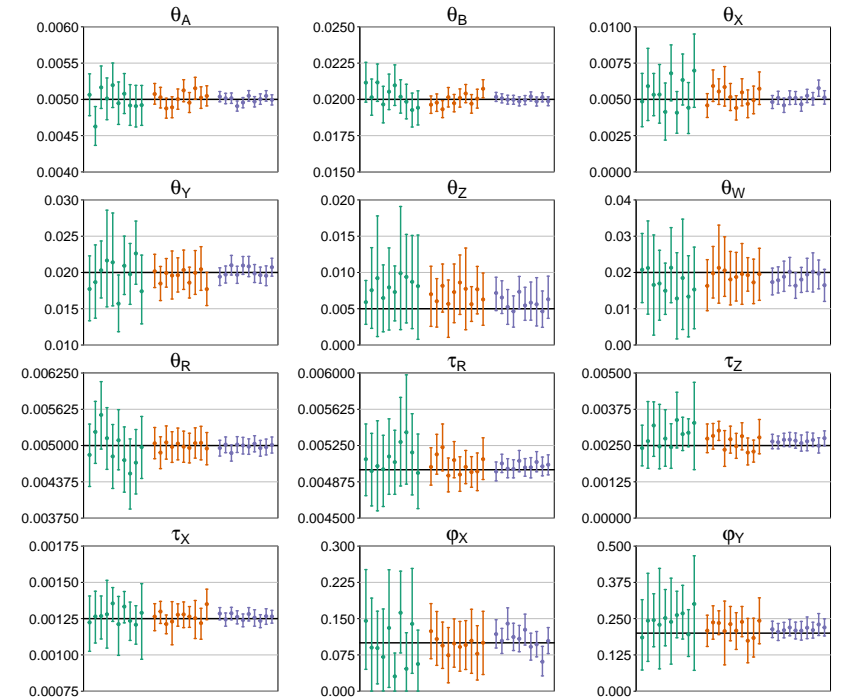

Q

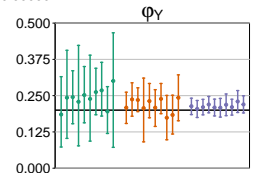

Fig. 8. Posterior means and the $95 \% \mathrm{HPD} \mathrm{Cls}$ in 10 replicate datasets of $L=$ 500,2000 , and 8000 loci, simulated and analyzed under the double-BDI model of figure $6 \mathrm{a}$. The MCMC samples are post-processed using the $\beta-\gamma$ algorithm before they are summarized. 
bioRxiv preprint doi: https://doi.org/10.1101/2021.08.14.456331; this version posted August 15,2021 . The copyright holder for this preprint (which was not certified by peer review) is the author/funder, who has granted bioRxiv a license to display the preprint in perpetuity. It is made available under aCC-BY-NC-ND 4.0 International license.

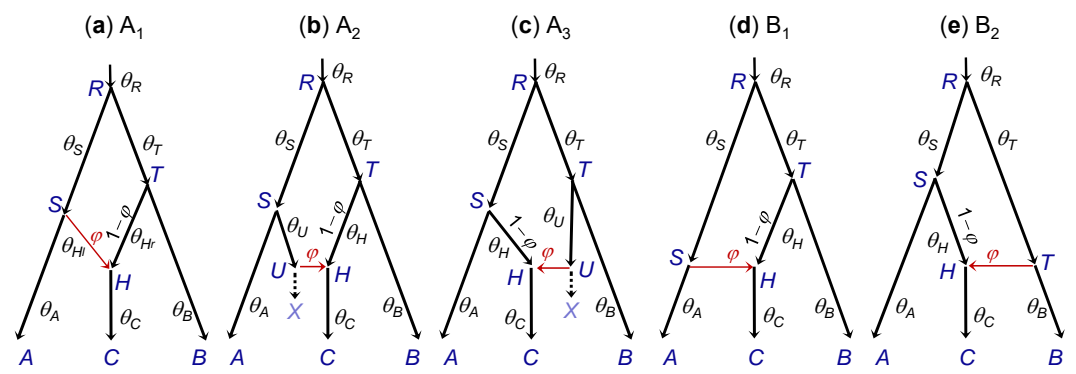

Fig. 9. (a-c)Three interpretations of MSci model A are indistinguishable/unidentifiable. (d, e) Two versions of MSci model B are identifiable. 
bioRxiv preprint doi: https://doi.org/10.1101/2021.08.14.456331; this version posted August 15, 2021. The copyright holder for this preprint (which was not certified by peer review) is the author/funder, who has granted bioRxiv a license to display the preprint in perpetuity. It is made available under aCC-BY-NC-ND 4.0 International license.

\section{Supporting Information (SI).}

- Figure S1: Analysis of the first 500 exonic loci of the Heliconius data.

- Figure S2: Three models with a BDI event between sister species.

- Figure S3: Two models with a BDI event between nonsister species.

- Figure S4: Three models with a BDI event between nonsister species.

- Figure S5: Two BDI events between non-sister species creating four unidentifiable models.

- Figure S6: Trace plots for $\phi_{X}$ and $\phi_{Y}$ in analysis of a dataset of $L=500$ loci simulated under the BDI model of figure 1.

- Table S1: Posterior means and 95\% HPD CIs for parameters in the BDI model from a simulated data of $L=500$ loci.
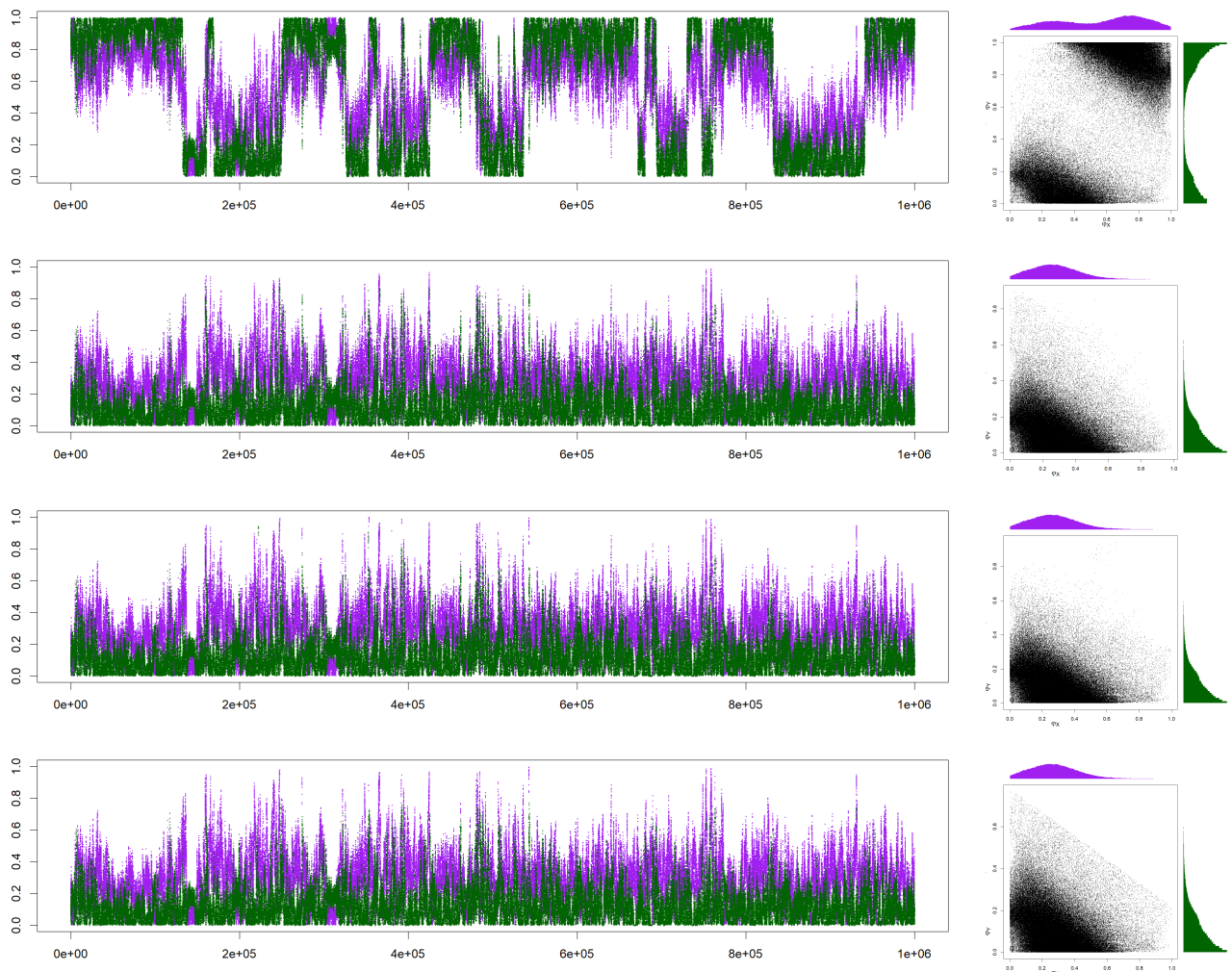

Fig. S1. Analysis of the first 500 exonic loci on chromosome 1 from the Heliconius data. See legend to figure 3. 
bioRxiv preprint doi: https://doi.org/10.1101/2021.08.14.456331; this version posted August 15, 2021. The copyright holder for this preprint (which was not certified by peer review) is the author/funder, who has granted bioRxiv a license to display the preprint in perpetuity. It is made available under aCC-BY-NC-ND 4.0 International license.

(a) Sister species $\Theta$
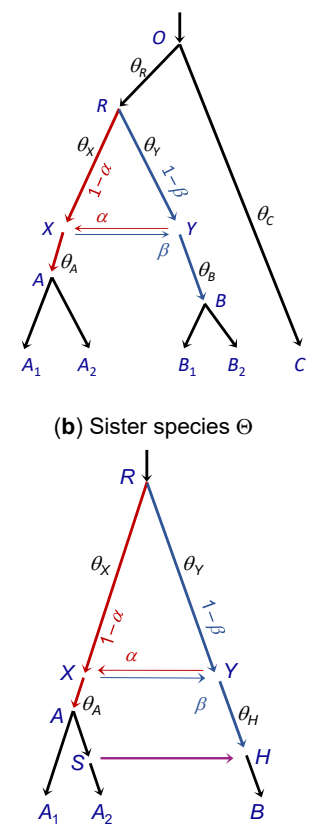

(c) Sister species $\Theta$

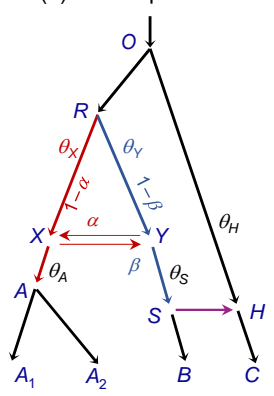

(a') Sister species, $\Theta^{\prime}$

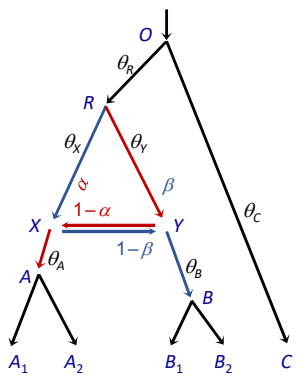

(b') Sister species, $\Theta^{\prime}$

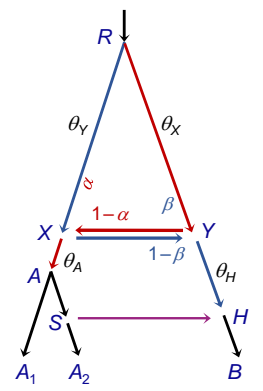

(c') Sister species, $\Theta^{\prime}$

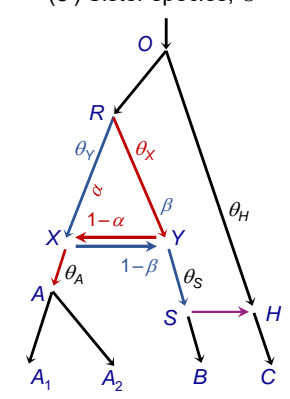

fig4:D-sister

Fig. S2. Three models, each involving a BDI event between sister species, creating within-model unidentifiability. (a) \& $\mathbf{a}^{\prime}$ ) Subtrees are added to branches $A, B$, and $R$ in the basic model of figure 1a. (b) \& $\mathbf{b}^{\prime}$ ) A BDI event between sister species $X$ and $Y$ with a unidirectional introgression involving descendant branches of $X$ and $Y$. (c) and $\left.\mathbf{c}^{\prime}\right)$ A BDI event between sister species $X$ and $Y$ with a unidirectional introgression involving one descendant branch and another branch that is not a descendant of $X$ or $Y$. In all three cases, the parameter mapping is $\varphi_{X}^{\prime}=1-\varphi_{X}, \varphi_{Y}^{\prime}=1-\varphi_{Y}, \theta_{X}^{\prime}=\theta_{Y}$, and $\theta_{Y}^{\prime}=\theta_{X}$.

\section{(a) Tree $S$ with $\Theta$}

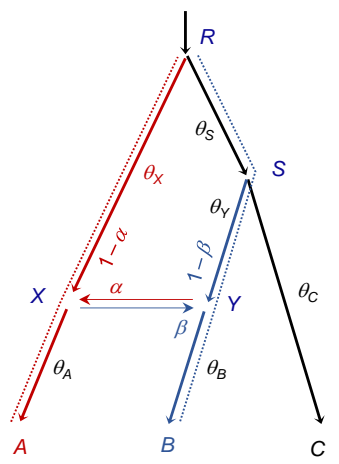

(b) Tree $S$ with $\Theta$

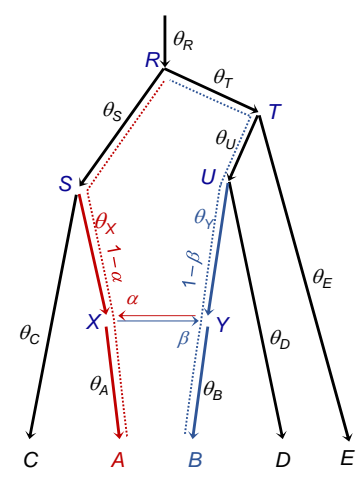

(a') Tree $S^{\prime}$ with $\Theta '$

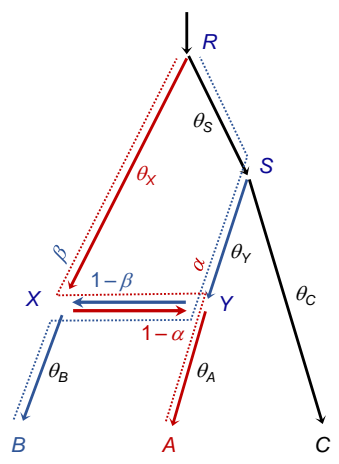

(b') Tree $S^{\prime}$ with $\Theta^{\prime}$

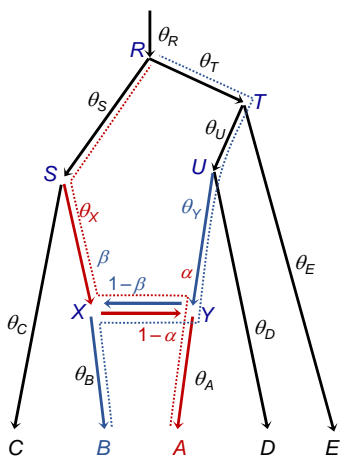

fig5:D-nonsister

Fig. S3. BDI between non-sister species creates cross-model unidentiability. (a \& $\left.\mathbf{a}^{\prime}\right)$ A pair of unidentifiable models with a BDI event between non-sister species. The dotted lines indicate the main routes taken by sequences sampled from species $A$ and $B$, if the introgression probabilities $\alpha$ and $\beta$ are $<\frac{1}{2}$. ( $\left(\mathbf{b} \& \mathbf{b}^{\prime}\right)$ Another pair of unidentifiable models with a BDI event between non-sister species. The parameter mapping from $\Theta$ to $\Theta^{\prime}$ in both cases is $\varphi_{X}^{\prime}=1-\varphi_{Y}$ and $\varphi_{Y}^{\prime}=1-\varphi_{X}$, with all other parameters (such as $\theta_{X}, \theta_{Y}, \theta_{A}$, and $\theta_{B}$ ) to be identical between $\Theta$ and $\Theta^{\prime}$. 
bioRxiv preprint doi: https://doi.org/10.1101/2021.08.14.456331; this version posted August 15, 2021. The copyright holder for this preprint (which was not certified by peer review) is the author/funder, who has granted bioRxiv a license to display the preprint in perpetuity. It is made available under aCC-BY-NC-ND 4.0 International license.

(a) Tree $S$ with $\Theta$

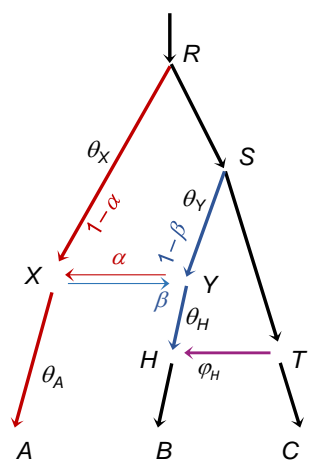

(b) Tree $S$ with $\Theta$

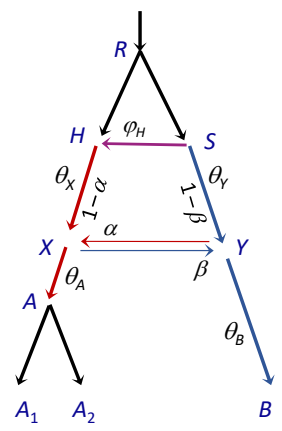

(c) Tree $S$ with $\Theta$

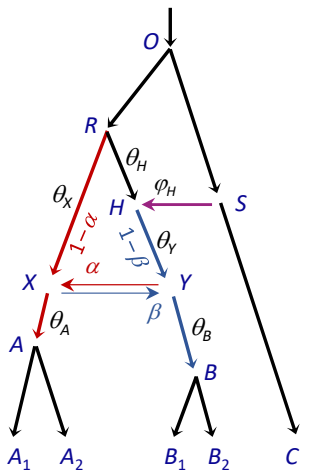

(a') Tree $S^{\prime}$ with $\Theta^{\prime}$

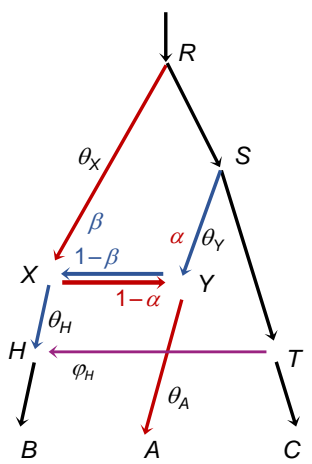

(b') Tree $S^{\prime}$ with $\Theta^{\prime}$

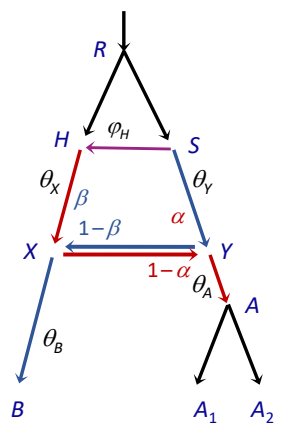

(c') Tree $S^{\prime}$ with $\Theta^{\prime}$

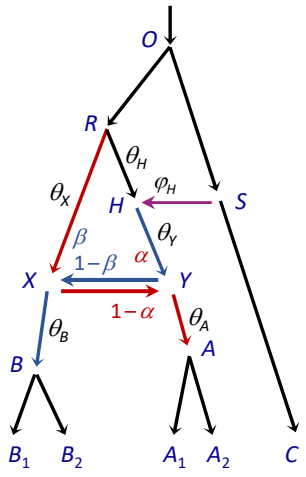

fig6:D-nonsister2

Fig. S4. Three pairs of unidentifiable models with one BDI event between non-sister species, illustrating the mapping of parameters $\left(\Theta\right.$ and $\left.\Theta^{\prime}\right)$. In (a), RXA and $S Y H$ are non-sister species. In (b \& c), nodes $X$ and $Y$ are non-sister species because of the unidirectional introgression event involving branches $R X$ and/or $R Y$. The mirror model $\left(S^{\prime}\right.$ with $\left.\Theta^{\prime}\right)$ is generated by pruning off branches $A X$ at $X$ and $B Y$ at $Y$, swapping places and reattaching, and applying the mapping $\varphi_{X}^{\prime}=1-\varphi_{Y}$ and $\varphi_{Y}^{\prime}=1-\varphi_{X}$.

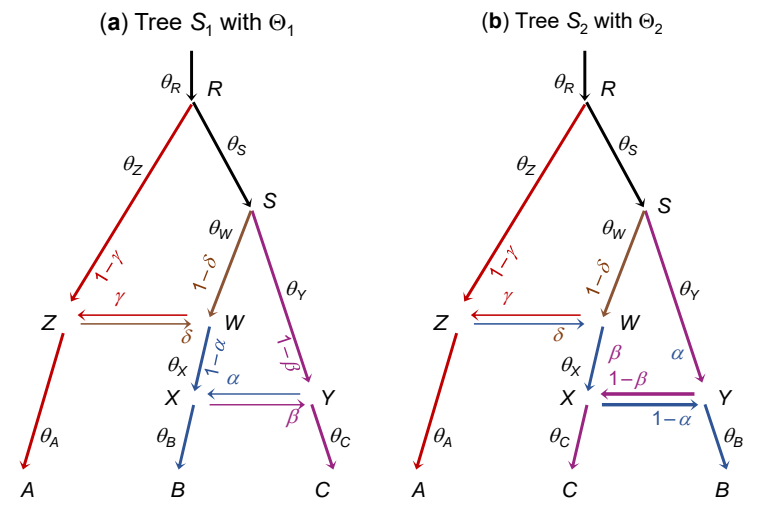

(c) Tree $S_{3}$ with $\Theta_{3}$

(d) Tree $S_{4}$ with $\Theta_{4}$

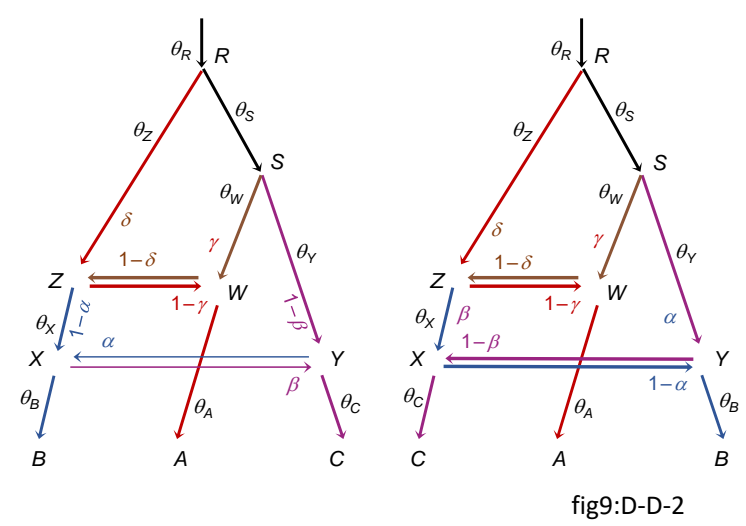

Fig. S5. Two BDI events involving non-sister species on a species tree for three species creating four unidentifiable models. The cross-model parameter mappings concern only the introgression probabilities $\varphi_{X} \equiv \alpha, \varphi_{Y} \equiv \beta, \varphi_{Z} \equiv \gamma$, and $\varphi_{W} \equiv \delta$, while all other parameters are the same among the models. The colored lines indicate the main routes taken by sequences sampled from $A$ (red), $B$ (blue), and $C$ (purple), if the introgression probabilities $\alpha, \beta, \gamma$, and $\delta$ are all $<\frac{1}{2}$, from which the unidentifiability of the four models can be seen easily. 
bioRxiv preprint doi: https://doi.org/10.1101/2021.08.14.456331; this version posted August 15, 2021. The copyright holder for this preprint (which was not certified by peer review) is the author/funder, who has granted bioRxiv a license to display the preprint in perpetuity. It is made available under aCC-BY-NC-ND 4.0 International license.
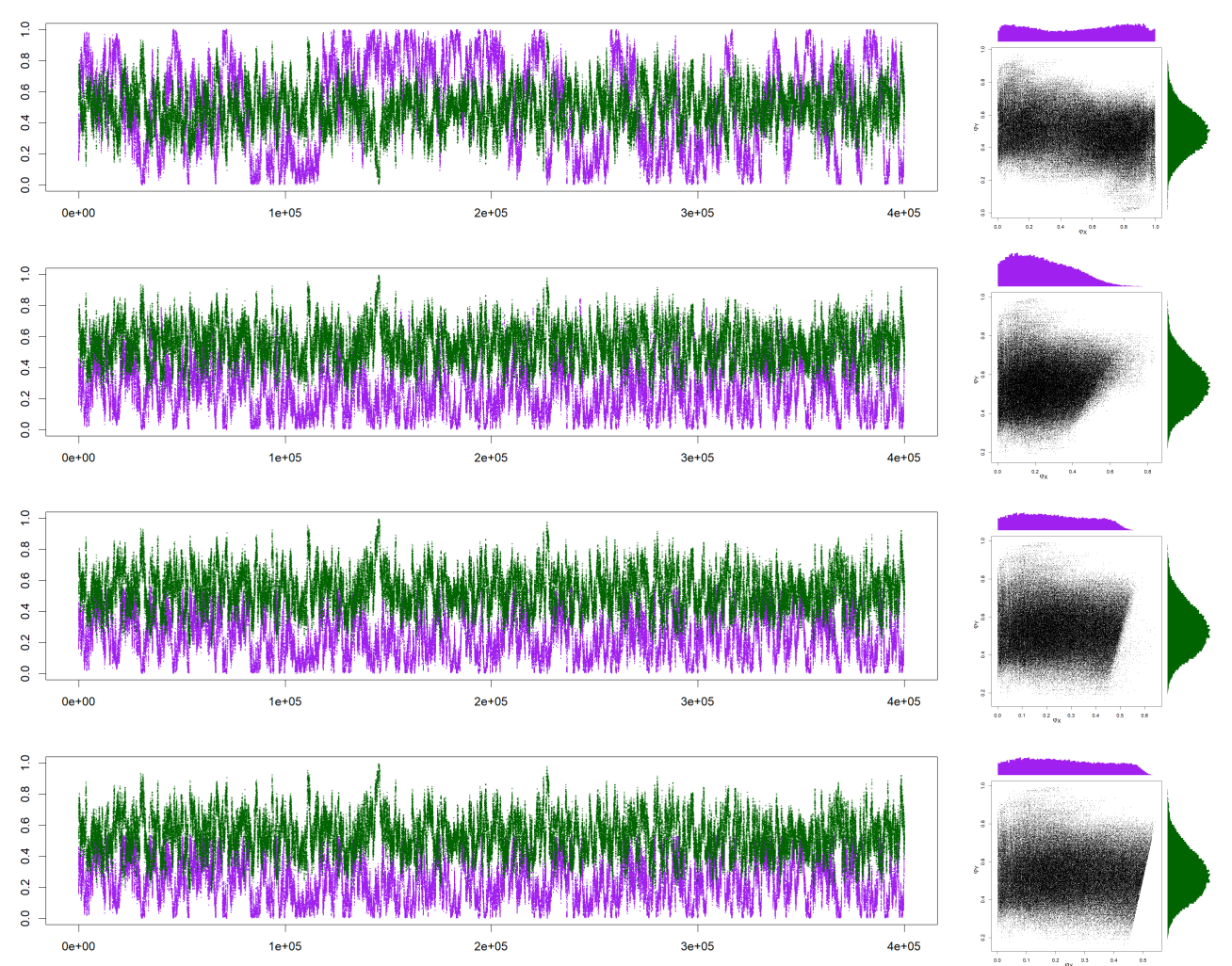

Fig. S6. Trace plots of MCMC samples for $\phi_{X}$ and $\phi_{Y}$ and 2-D scatter plots from BPP analysis of a dataset of $L=500$ loci simulated under the BDI model of figure 1 a. See table $\mathrm{S} 1$ for the true parameter values and posterior summaries. The plots are for, from top to bottom, unprocessed sample and processed samples using the $\beta-\gamma$, CoG ${ }_{N}$, and $\mathrm{CoG}_{0}$ algorithms. The true parameter values are $\Theta=\left(\varphi_{X}, \varphi_{Y}\right)=(0.7,0.2)$, and the post-processing using all three algorithms mapped the samples to the mirror tower around $\Theta^{\prime}=(0.3,0.8)$.

Table S1. Posterior means and 95\% HPD Cls (in parenthees) for parameters in the MSci model of figure 1a from a simulated dataset of $L=500$ loci

\begin{tabular}{lllccc}
\hline & truth $(\Theta)$ & mirror $\left(\Theta^{\prime}\right)$ & beta-gamma & $\operatorname{CoG}_{N}$ & $\operatorname{CoG}_{0}$ \\
\hline$\tau_{R}$ & 0.01 & & $0.0098(0.0088,0.0108)$ & & \\
$\tau_{X}=\tau_{Y}$ & 0.005 & & $0.0050(0.0045,0.0055)$ & & \\
$\theta_{A}$ & 0.002 & & $0.0020(0.0018,0.0021)$ & & \\
$\theta_{B}$ & 0.01 & & $0.0101(0.0093,0.0108)$ & & \\
$\theta_{R}$ & 0.002 & & $0.0020(0.0006,0.0034)$ & & \\
$\theta_{X}$ & 0.002 & 0.01 & $0.0071(0.0022,0.0124)$ & $0.0067(0.0017,0.0120)$ & $0.0068(0.0017,0.0121)$ \\
$\theta_{Y}$ & 0.01 & 0.002 & $0.0063(0.0005,0.0130)$ & $0.0066(0.0005,0.0133)$ & $0.0066(0.0005,0.0133)$ \\
$\phi_{X}$ & 0.7 & 0.3 & $0.245(0.001,0.528)$ & $0.236(0.001,0.472)$ & $0.235(0.001,0.470)$ \\
$\phi_{Y}$ & 0.2 & 0.8 & $0.553(0.330,0.791)$ & $0.539(0.305,0.786)$ & $0.538(0.305,0.788)$ \\
\hline
\end{tabular}

Note.- Empty cells mean the same values as on the left. MCMC samples are processed using the three algorithms and then summarized. See figure S6 for the tracecatter plots. The dataset of $L=500$ loci, each consisting of four sequences per species (or eight sequences per locus) and 500 sites per sequence, is simulated using the true parameter values $(\Theta)$. 